\title{
Strong earthquake activity all over the world and strong-moderate earthquake ac- tivity within and near China (October 2007 November 2007)
}

\author{
CHEN Pei-shan (陈培善) \\ Institute of Geophysics, China Earthquake Administration, Beijing 100081, China \\ CLC number: P315.5 Document code: $\mathrm{R}$
}

\section{Illustration}

All the data in this catalog are chosen from the "Preliminary Seismological Report of Chinese Seismic Stations" (Its abbreviation is "Monthly Report"). The catalog includes the events of $M \geq 4.7$ in and near China and $M \geq 6$ all over the world. The "Monthly Report" is monthly compiled by the Ninth Section of Institute of Geophysics, China Earthquake Administration.

The origin times of earthquakes in the catalog adopt coordinated universal time (UTC) in accordance with international convention. The location of every epicenter is expressed by its latitude and longitude, at the same time by the corresponding geographical region proposed by Flinn and Engdahl. The regional names are only for references and do not imply any political significance. The number of stations used and standard deviation are also given in order to illustrate the precision of location.

The surface wave magnitude $M_{\mathrm{S}}$ is measured from the records of intermediate period broad band SK seismographs and adopting the surface wave magnitude formula of Beijing Station of 1965: $M_{\mathrm{S}}=\lg \left(A_{\mathrm{H}} / T\right)+1.66 \lg \Delta+3.5\left(1^{\circ}<\Delta<130^{\circ}\right)$, in which $A_{\mathrm{H}}$ is the resultant displacement amplitude of the maximum surface wave of horizontal components. $M_{\mathrm{S} 7}$ is measured from records of the long-period seismographs of 763 type and adopting the surface wave magnitude formula recommended by IASPEI in 1967. $M_{\mathrm{S} 7}=\lg \left(A_{\mathrm{V}} / T\right)+1.66 \lg \Delta+3.3\left(20^{\circ}<\Delta<160^{\circ}\right)$, in which $A_{\mathrm{V}}$ is the maximum ground displacement of surface wave in vertical component, $m_{\mathrm{b}}$ is short-period body-wave magnitude. $M_{\mathrm{L}}$ is local magnitude. In order to avoid confusion, no conversion is made among the various magnitudes. For convenience of use and comparison, the surface wave magnitude $M_{\mathrm{SZ}}$ (NEIS) and $m_{\mathrm{b}}$ (NEIS) measured by NEIS recorded on short period seismographs are also listed. 
Catalog of earthquakes within and near China

(October 2007 November 2007; $M \geq 4.7$ )

\begin{tabular}{|c|c|c|c|c|c|c|c|c|c|c|c|c|c|c|}
\hline \multirow{3}{*}{ No. } & \multirow{2}{*}{\multicolumn{2}{|c|}{$\begin{array}{l}\text { Origin time } \\
\text { (UTC) }\end{array}$}} & \multirow{2}{*}{\multicolumn{2}{|c|}{$\begin{array}{l}\text { Geographic } \\
\text { coordinates }\end{array}$}} & \multirow{3}{*}{$\begin{array}{c}\text { Focal } \\
\text { depth } \\
/ \mathrm{km}\end{array}$} & \multicolumn{6}{|c|}{ Magnitudes } & \multirow{3}{*}{ SD } & \multirow{3}{*}{$\begin{array}{l}\text { No. } \\
\text { sta. } \\
\text { used }\end{array}$} & \multirow{3}{*}{ Region } \\
\hline & & & & & & \multicolumn{4}{|c|}{ China } & \multicolumn{2}{|c|}{ NEIS } & & & \\
\hline & $\mathrm{d}$ & h:min:s & $\varphi_{\mathrm{N}} /^{\circ}$ & $\lambda_{\mathrm{E}} /^{\circ}$ & & $M_{\mathrm{S}}$ & $M_{\mathrm{S} 7}$ & $M_{\mathrm{L}}$ & $m_{\mathrm{b}}$ & $M_{\mathrm{SZ}}$ & $m_{\mathrm{b}}$ & & & \\
\hline \multicolumn{15}{|c|}{ October 2007} \\
\hline 1 & 5 & $14: 24: 45.7$ & 43.88 & 130.88 & 571 & & & & 4.8 & & 4.3 & 0.7 & 45 & E.Russia-N.E.China border region \\
\hline 2 & 9 & $16: 00: 43.4$ & 43.01 & 77.94 & 28 & 4.5 & 4.3 & 4.9 & 4.7 & & 5.0 & 1.6 & 52 & Alma-Ata region \\
\hline 3 & 14 & $01: 05: 33.7$ & 37.74 & 76.15 & 21 & 4.0 & 3.7 & 4.8 & 4.8 & & 4.3 & 2.4 & 24 & Tadzhikistan-Xinjiang border region \\
\hline 4 & 16 & $06: 46: 57.3$ & 20.31 & 100.87 & 30 & 4.7 & 4.3 & 4.8 & 4.5 & & 4.6 & 1.7 & 33 & Indo-Pacific Peninsula \\
\hline 5 & 17 & $14: 40: 0.2$ & 23.68 & 121.58 & 41 & 4.6 & 4.4 & 5.0 & 4.6 & 4.5 & 5.1 & 1.5 & 75 & Taiwan \\
\hline 6 & 26 & $06: 50: 05.7$ & 35.30 & 76.80 & 10 & 5.1 & 4.9 & 5.6 & 5.1 & 4.8 & 5.3 & 1.3 & 73 & Eastern Kashmir \\
\hline 7 & 29 & $09: 49: 12.6$ & 27.34 & 85.00 & 10 & 4.7 & 4.4 & & 4.6 & & 4.7 & 1.9 & 26 & Nepal-India border region \\
\hline 8 & 30 & 09:20:03.6 & 33.21 & 90.36 & 25 & 4.3 & 3.8 & & 4.7 & & 3.7 & 3.4 & 13 & Tibet \\
\hline \multicolumn{15}{|c|}{ November 2007} \\
\hline 9 & 6 & $02: 15: 47.2$ & 38.54 & 73.25 & 121 & & & & 4.9 & & 5.0 & 1.5 & 58 & Tadzhikistan \\
\hline 10 & 7 & $22: 54: 14.3$ & 25.08 & 122.37 & 16 & 4.6 & 4.4 & 4.8 & 4.3 & & 4.6 & 2.2 & 36 & Taiwan \\
\hline 11 & 10 & $19: 36: 23.1$ & 29.48 & 95.42 & 25 & 4.3 & 4.1 & 4.5 & 4.9 & 4.0 & 5.0 & 2.2 & 65 & India-China border region \\
\hline 12 & 13 & $05: 57: 35.1$ & 37.13 & 80.35 & 15 & 4.4 & 4.2 & 4.8 & 4.3 & 4.2 & 4.9 & 2.8 & 25 & Southern Xinjiang Province \\
\hline 13 & 17 & $10: 08: 14.5$ & 39.29 & 119.12 & 18 & 3.6 & 3.6 & 3.7 & 4.7 & & & 2.2 & 15 & North-Eastern China \\
\hline 14 & 23 & $11: 17: 11.6$ & 25.06 & 122.40 & 18 & 4.9 & 4.6 & & 4.4 & & 4.5 & 2.5 & 9 & Taiwan \\
\hline 15 & 23 & $11: 20: 09.1$ & 24.80 & 122.50 & 35 & 4.7 & 4.6 & & 4.3 & & 4.5 & 3.9 & 8 & Taiwan region \\
\hline 16 & 24 & $19: 33: 50.5$ & 25.31 & 124.82 & 120 & & & & 4.7 & & 4.5 & 0.7 & 8 & South-western Ryukyu Islands \\
\hline 17 & 28 & $21: 05: 11.9$ & 24.85 & 122.06 & 62 & 4.1 & 4.1 & 4.7 & 4.4 & & 4.6 & 1.6 & 45 & Taiwan \\
\hline
\end{tabular}

Catalog of earthquakes all over the world

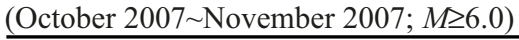

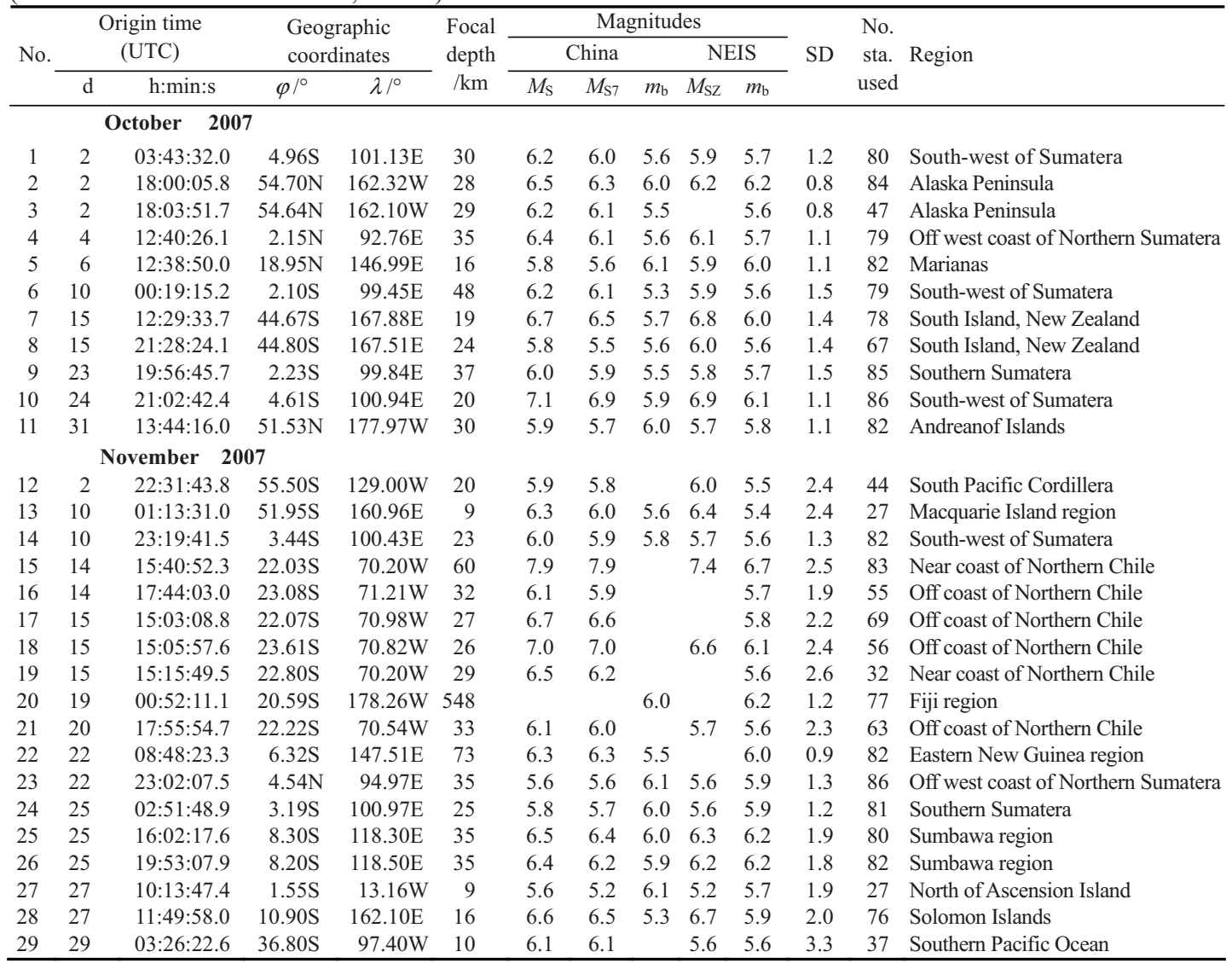

